\title{
Supporting Information: Hydrogen Dynamics in Supercritical Water Probed by Neutron Scattering and Computer Simulations
}

Carla Andreani, ${ }^{\dagger} \ddagger$ Giovanni Romanelli, ${ }^{,}$- Alexandra Parmentier, ${ }^{\S}$ Roberto Senesi, ${ }^{\dagger, \ddagger}$ Alexander I. Kolesnikov, $\|$ Hsin-Yu Ko, ${ }^{\perp, \#}$ Marcos F. Calegari Andrade, ${ }^{\perp}$ and Roberto Car $^{\circledR}$

$\dagger$ †niversità degli Studi di Roma Tor Vergata, Dipartimento di Fisica and NAST Centre, Via della Ricerca Scientifica 1, 00133, Roma, Italy

$\ddagger C N R$-IPCF, Istituto per i Processi Chimico-Fisici del CNR di Messina, Viale F. Stagno dAlcontres 37, 98158 Messina, Italy

ฯISIS Facility, Rutherford Appleton Laboratory, Chilton, Didcot, Oxfordshire, OX11 0QX, United Kingdom

$\S I N F N$ - Division of Rome Tor Vergata, via della Ricerca Scientifica 1, 00133, Rome, Italy \|Neutron Scattering Division, Oak Ridge National Laboratory, Oak Ridge, Tennessee 37831, United States of America

$\perp$ Department of Chemistry, Princeton University, Princeton, New Jersey 08544, USA \#Present address: Department of Chemistry and Chemical Biology, Cornell University, Ithaca, New York 14580, USA

@ Department of Chemistry and Department of Physics, Princeton University, Princeton, New Jersey 08544, USA

E-mail: giovanni.romanelli@stfc.ac.uk 


\section{Supporting Information}

\section{INS measurements}

INS measurements were performed on the SEQUOIA spectrometer ${ }^{1}$ (SNS, Oak Ridge National Laboratory, US) in order to access the nuclear vibrational densities of states via thermal neutron scattering on a mostly incoherent sample. In the incoherent approximation, the water dynamic structure factor $S(Q, E)$ is dominated by the hydrogen contribution, and can be directly related to the hydrogen-projected vibrational density of states, $g_{\text {exp }}(E)$, via the relation

$$
S_{\text {inc }}(Q, E)=\frac{N_{H}}{2 M} Q^{2} \frac{g_{\text {exp }}(E)}{E}[n(E)+1] e^{-2 W(Q)},
$$

where $Q$ and $E$ are the momentum and energy transferred from the neutron to the system, $N_{H}$ and $M$ are the number and mass of $\mathrm{H}$ atoms, $n(E)$ is the Bose-Einstein population factor $n(E)=1 /\left[\exp \left(E / k_{B} T\right)-1\right], k_{B}$ is the Boltzmann constant, and $2 W(Q)$ is the DebyeWaller factor. Experiments were performed on a bulk $\mathrm{H}_{2} \mathrm{O}$ sample loaded in 38 cylindrical holes (diameter $=1 \mathrm{~mm}$ ) within a Ti container. An aluminum wrapping at temperatures larger than $733 \mathrm{~K}$ was added in order to prevent thermal dispersion. The dimensions of the container were $(5 \mathrm{~cm}) \times(5 \mathrm{~cm}) \times(2.03 \mathrm{~mm})$. Measurements were performed following an isobaric line at $P=250$ bar (higher than $P_{C}=221$ bar) and in the temperature range 553 $\mathrm{K} \leq T \leq 823 \mathrm{~K}$, that is, from the sub-critical region $\left(T<T_{C}=647 \mathrm{~K}\right)$ to the super-critical (SC) region. SEQUOIA is a direct-geometry INS spectrometer, with incident energy selected through a chopper at $E_{i}=800 \mathrm{meV}$ for the present experiment. Spectra for each detector were corrected for the empty-container background using the MANTID software routines available for the instrument. Spectra from detectors sharing the same scattering angle $\theta$ within 6 degrees were grouped together to improve signal-to-noise ratio.

Multiple-scattering (MS) corrections were modelled analytically simulating only doublescattering with $\theta \sim 90$ degrees for each scattering event. This model has been applied in the past, ${ }^{2,3}$ and has been proven successful for spectra at angles lower than 25 degrees. The MS 
contribution in this approach is defined as

$$
M S(E)=\frac{A}{\sqrt{2 \pi} \sigma_{M S}} \exp \left[-\frac{\left(E-E_{M S}\right)^{2}}{2 \sigma_{M S}^{2}}\right]
$$

where $E_{M S}=2 E_{i}$. The MS Gaussian contribution was fitted to the experimental data in the region $600 \mathrm{meV} \leq E \leq 770 \mathrm{meV}$, with $A$ and $\sigma_{M S}$ used as fit parameters. Corrected spectra were expressed as functions of $Q$ and the density of vibrational states was obtained using the procedure

$$
g_{\text {exp }}(E)=\lim _{Q^{2} \rightarrow 0} \frac{S_{i n c}(Q, E)}{Q^{2}} 2 M E \frac{e^{2 W(Q)}}{n(E)+1} .
$$

In the case of the low-scattering angles considered here, $g_{\exp }(E)$ was obtained for each energy channel via a linear regression applied to the points at $\theta=6,12,18$ and 24 degrees.

\section{DINS measurements}

DINS Experiments were performed on the VESUVIO spectrometer (ISIS pulsed neutron and muon source, Rutherford Appleton Laboratory, UK). VESUVIO is an inverted-geometry spectrometer with final energy $E_{f} \sim 4.9 \mathrm{eV}$ and initial energy in the epithermal energy region up to hundreds of $\mathrm{eV}$. DINS studies involve energy transfers in excess of $1 \mathrm{eV}$ and momentum transfers in excess of $20 \AA^{-14-6}$ and scattering is recorded by neutron and gamma detectors. ${ }^{7-12}$ As the energy transfer is much higher than any binding energy, and the inverse of momentum transfer is much smaller than any interparticle distance in the system, DINS formalism is expressed within the Impulse Approximations. In particular, the dynamic structure factor can be expressed as

$$
S_{I A}(Q, E)=\sum_{M} \int n(p) \delta\left(\frac{E-E_{r}}{\hbar}-\frac{\vec{p} \cdot \vec{Q}}{M}\right) d \vec{p}=\sum_{M} \frac{M}{\hbar Q} J\left(y_{M}\right)
$$

where $y_{M}=\vec{p} \cdot \hat{Q} / \hbar$ is the projection of the momentum $\vec{p}$ of an atom of mass $M$ along the $\vec{Q}$ direction, and $J\left(y_{M}\right)$ is the neutron Compton profile NCP, ${ }^{13}$ and the sum runs over all the 
isotopic species in the sample. The dependence of $y_{M}$ on the mass of the scattering atom makes DINS a unique mass-selective neutron spectroscopy. Figure S1 shows DINS spectra expressed as functions of the $y_{H}$ variable, where the separation of the hydrogen signal from those related to the heavier O and Ti masses is evident. The shape of the NCP is related

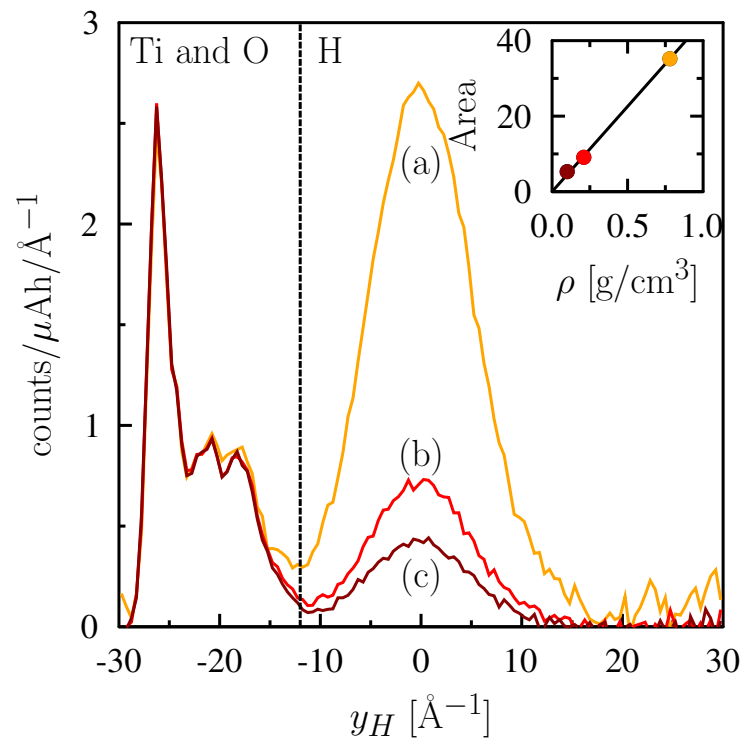

Figure S1: DINS data (as the sum over front scattering detectors) at $T=553 \mathrm{~K}$ (a, orange line); $663 \mathrm{~K}$ (b, red line); $733 \mathrm{~K}$ (c, dark-red line). The dotted vertical line at $y_{H}=-12$ $\AA^{-1}$ is the lower cut-off for the peak area integration reported in the inset.

to the nuclear momentum distribution $n(\vec{p})$ of the target atom and it is broadened mainly because of the zero-point energy (ZPE) of the atom in its confining potential. The second moment $\sigma^{2}=\left\langle y^{2}\right\rangle$ of the $\mathrm{NCP}$ is directly related to the atom mean kinetic energy

$$
\left\langle E_{K}\right\rangle=\frac{3 \hbar^{2} \sigma^{2}}{2 M}=3 \int_{0}^{\infty} \frac{\hbar \omega}{4} g(\omega) \operatorname{coth}\left(\frac{\hbar \omega}{2 k_{B} T}\right) d \omega,
$$

as a consequence of the Virial theorem. Moreover, the intensity of the peak is directly proportional (in the single-scattering limit) to the number of atoms weighted by their neutron bound scattering cross section. As an example, looking at Figure S1 one can notice how the intensity of the hydrogen peak falls as the temperature goes from $553 \mathrm{~K}$ (a), to $663 \mathrm{~K}$ (b) and $773 \mathrm{~K}$ (c) and the water density decreases according to Table S1. Measurements were performed along the same isobaric line as the INS experiment, and using the same Ti container. The linear dependence of the intensity of the hydrogen NCP on the water density 
Table S1: Thermodynamic variables corresponding to INS and DINS measurements presented in this work.

\begin{tabular}{cccc}
$\mathrm{T}[\mathrm{K}]$ & $\mathrm{P}[\mathrm{bar}]$ & $\rho[\mathrm{g} / \mathrm{ml}]$ & Technique \\
\hline \hline 553 & 250 & 0.777 & INS/DINS \\
623 & 250 & 0.625 & INS \\
663 & 250 & 0.215 & INS/DINS \\
733 & 250 & 0.104 & INS/DINS \\
823 & 250 & 0.078 & INS \\
\hline \hline
\end{tabular}

(proportional to the number of hydrogen atoms hit by the neutron beam) is reported in the inset included in the same Figure, and suggests a small contribution from multiple scattering in the case of DINS data. Time-of-flight spectra recorded by VESUVIO front-scattering detectors were first corrected by sample-dependent and environmental backgrounds following standard procedures. Single and multiple scattering were reproduced by an ad hoc Monte Carlo simulation, while MS and single-scattering contributions from masses heavier than $\mathrm{H}$ were subtracted from experimental spectra. Each spectrum was then transformed from the time-of-flight to the $y_{H}$ domain, where a global fit was performed having the width of the NCP as a global parameter. Deviations from the IA, $\Delta J\left(y_{H}, Q\right)$, were corrected as additive contributions to $J\left(y_{H}\right)$ proportional to its third-derivative. Such contributions hold a Qdependency spoiling the possibility to analyse the sum of the spectra from all detectors (as in Figure S1), therefore requiring the global fit to be performed. The resulting model function was numerically convoluted with the simulated instrument resolution for the spectrum from each detector $l$, so as to obtain the fitted function $F\left(y_{H}, Q\right)$

$$
F_{l}\left(y_{H}, Q\right)=\left[J_{I A}\left(y_{H}\right)+\Delta J\left(y_{H}, Q\right)\right] \star R_{l}\left(y_{H}\right)
$$

\section{Density Functional Theory Calculations}

Electronic structure calculations to construct the DP model were performed with the opensource code Quantum ESPRESSO (QE). ${ }^{14,15}$ We adopted the strongly-constrained-and-appropriately- 
normed (SCAN) ${ }^{16}$ meta-generalized-gradient approximation (meta-GGA) for the exchangecorrelation energy and used a cutoff of 110 Ry in the plane-wave expansion of the pseudowavefunctions. SCAN has been shown to provide improved structural and dynamical description of water systems compared with conventional GGA methods. ${ }^{17,18}$ The interaction of the valence electrons with the nuclei plus frozen core electrons was described by Hamann-Schlüter-Chiang-Vanderbilt norm-conserving pseudopotentials ${ }^{19,20}$ generated at the Perdew-Burke-Ernzerhof (PBE) GGA level of theory ${ }^{21}$ and distributed with the Qbox package. $^{22}$ Since we did not use SCAN pseudopotentials our calculations are not fully consistent, but we checked that this inconsistency has negligible effect on the binding properties. Valence-only SCAN calculations with PBE pseudopotentials give a mean absolute error of $\approx 0.4 \mathrm{meV} /$ atom, relative to all-electron SCAN calculations, for the binding energy in the S22 molecular database. ${ }^{23}$ This error should be attributed to the pseudopotential approximation itself, rather than to the (slightly) inconsistent pseudopotentials, as it is approximately the same error that we find when comparing valence-only and all-electron calculations using consistently the PBE functional. Static DFT calculations (electronic minimizations) were performed at the atomic configurations labelled by DP-GEN. As discussed in the main text, the construction of a DP model that reproduced accurately DFT at $p=250$ bar within the desired temperature range required a total of 3816 calculations at configurations labelled at $T=553 \mathrm{~K}$ and $T=623 \mathrm{~K}$, and a total of 2300 calculations at configurations labeled at $T=663 \mathrm{~K}, T=733 \mathrm{~K}$, and $T=823 \mathrm{~K}$. The calculations at the two lowest temperatures used a periodic 32-molecule cell, while those at the three highest temperatures sampled configurations having considerably lower atomic density and used a periodic 8-molecule cell. To further validate the model, two independent constant pressure $(N p T)$ Born-Oppenheimer AIMD trajectories were generated at $p=250$ bar and $T=553 \mathrm{~K}$ and $T=733 \mathrm{~K}$, respectively, with similar electronic structure and cell parameters of the corresponding static DFT calculations. Overall, the $T=553 \mathrm{~K}$ trajectory was $\approx 20 \mathrm{ps}$ long and the trajectory at $T=733 \mathrm{~K}$ was $\approx 40 \mathrm{ps}$ long. In these simulations, the deuterium mass was used for 
hydrogen in order to adopt a larger time step in the Verlet integrator. The temperature was controlled by a 4 -bead Nose-Hoover thermostat chain ${ }^{24}$ coupled to each atomic species, while the pressure was kept fluctuating around 250 bar by an isotropic Parrinello-Rahman barostat. $^{25}$

\section{Deep Potential Validation}

To validate the DP model, we compared the forces generated by the model with the corresponding DFT forces at the configurations visited in the AIMD trajectories discussed in the previous section. The AIMD configurations were not included in the fitting of the neural network, which was based on independent configurations labeled by DP-GEN. Yet, the DP forces were found to agree well with the AIMD forces, as illustrated in Figure S2. As noted in the main text, the standard deviation of the difference between DP and AIMD forces was less than $0.063 \mathrm{eV} / \AA$, which is less than $10 \%$ of the standard deviation of the AIMD atomic force distribution. The DP model was used to simulate water on $1 \mathrm{~ns}$ long trajectories for cells that are 27 times bigger than those of the corresponding AIMD simulations.

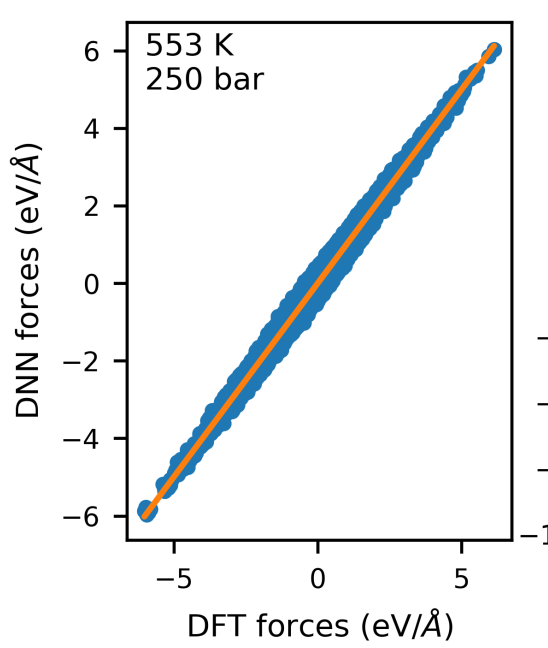

DFT forces $(\mathrm{eV} / \AA)$

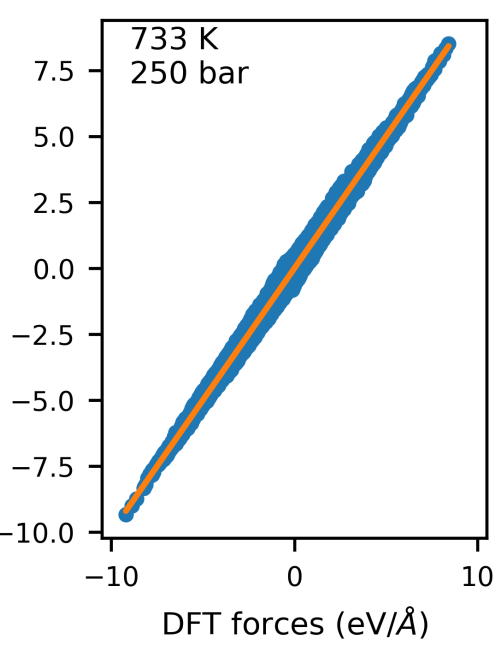

Figure S2: Comparison of the forces from DP and DFT for water at 250 bar and $553 \mathrm{~K}$ (left) and $733 \mathrm{~K}$ (right). 


\section{Hydrogen Bond Distortion}

The HBs become increasingly more distorted going from ambient condition (310 K, 1 bar) to subcritical (553 K, 250 bar) and supercritical (733 K, 250 bar) conditions as illustrated in Fig. S3. In this figure, we used the HB angle ( $\beta$ as depicted in the inset) following the Luzar-Chandler definition. ${ }^{26}$ Since this HB definition requires $\beta<30^{\circ}$, we also cut off the angular distributions at this value. Beyond this artificial angular cutoff, we observed even more distorted structures in supercritical water with bifurcated and cyclic HBs (Fig. S4). We note that even though the HB angle distribution depends on the HB definition and slightly different distributions would correspond to the different HB definitions considered in Tables S2 and S3, the trend of increasing HB distortion is robust going from ambient to subcritical and then to supercritical conditions.

In spite of the fact that the Luzar-Chandler definition misses the most distorted HB configurations at $733 \mathrm{~K}$, such as those shown in Fig. S4, this limitation of the HB definition has little effect on the non-percolating character of the HB network under this thermodynamic condition. We assess the extent of this effect by lifting the angular cutoff in the LuzarChandler definition, but keeping the radial cutoff at $3.5 \AA$. Even using this significantly more permissive definition (which also falsely includes a significant portion of non-hydrogenbonded configurations to water clusters), the HB network in the DPMD trajectory at $733 \mathrm{~K}$ remains completely non-percolating. This feature is fully consistent with the gas-like character of the pair correlation function in Fig. S5.

\section{Hydrogen-Bond Percolation Analysis}

We analyzed the HB network in the DPMD simulations using several standard HB definitions. While $N_{H B}$ fluctuates between 2.0 and 2.7, depending on the HB definition, in the fluid at $553 \mathrm{~K}$, we found that $N_{H B} \approx 0.4$ for all adopted $\mathrm{HB}$ criteria at $733 \mathrm{~K}$. We also performed cluster/percolation analysis on these two trajectories. At $553 \mathrm{~K}$, we took snapshots 


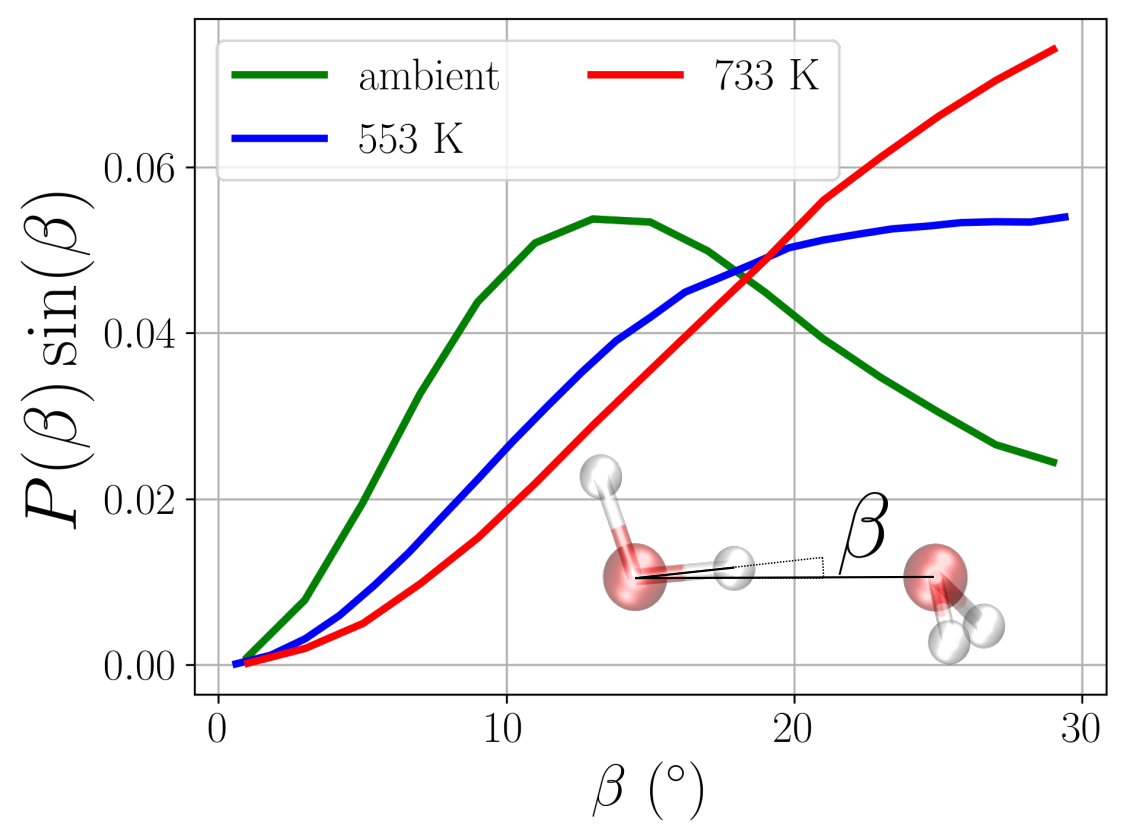

Figure S3: HB angle distributions of our DPMD simulations under ambient condition in green at (310 K, 1 bar), under experimental subcritical condition in blue at $(553 \mathrm{~K}, 250 \mathrm{bar})$, and under experimental supercritical condition in red at $(733 \mathrm{~K}, 250$ bar). The Luzar-Chandler HB definition was used. ${ }^{26}$ The HB angle is represented by $\beta$, as illustrated in the inset. The HB angle distributions were cutoff at $30^{\circ}$ following the HB definition and normalized such that $\int \mathrm{d} \beta P(\beta) \sin (\beta)=1$.

with an $\approx 1$-ps stride along the DPMD trajectory for HB analysis; for each snapshot we were always able to find a percolating cluster (see Table S2). On the other hand, at $733 \mathrm{~K}$, the HB network is always broken and the dominant configurations include monomers and small non-percolating oligomers (see Table S3).

While cluster and percolation analyses can depend on the HB definition, ${ }^{27}$ the percolation analysis turned out robust across a wide variety of HB definitions, as shown in Tables S2 and S3.

Table S2: Averaged number of HB, and fraction of percolating configuration $\left(f_{p}\right)$ in DPMD simulation at $553 \mathrm{~K}$

\begin{tabular}{cccccc} 
& $\begin{array}{c}\text { Luzar- } \\
\text { Chandler }\end{array}$ & $\begin{array}{c}\text { Todorova } \\
\text { et al. }\end{array}$ & Luzar $^{29}$ & $\begin{array}{c}\text { Kuo- } \\
\text { Mundy }^{30}\end{array}$ & $\begin{array}{c}\text { Wernet } \\
\text { et al. }^{31}\end{array}$ \\
\hline \hline$N_{H B}$ & 2.6 & 2.7 & 2.4 & 2.0 & 2.1 \\
$f_{p}$ & $100 \%$ & $100 \%$ & $100 \%$ & $100 \%$ & $100 \%$ \\
\hline \hline
\end{tabular}


a)

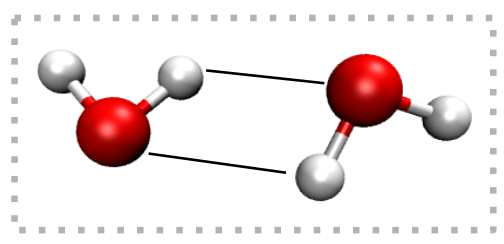

b)

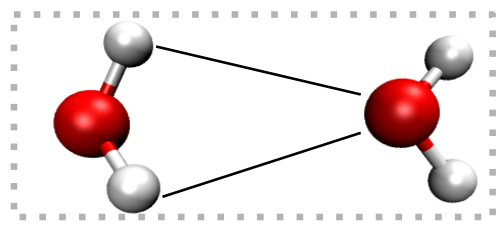

c)

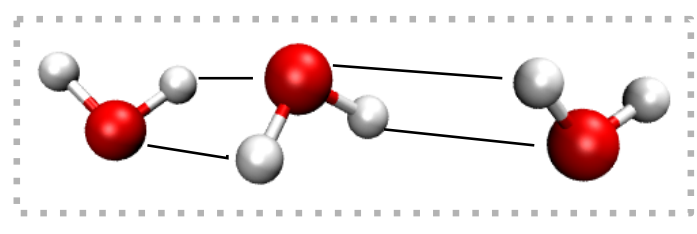

d)

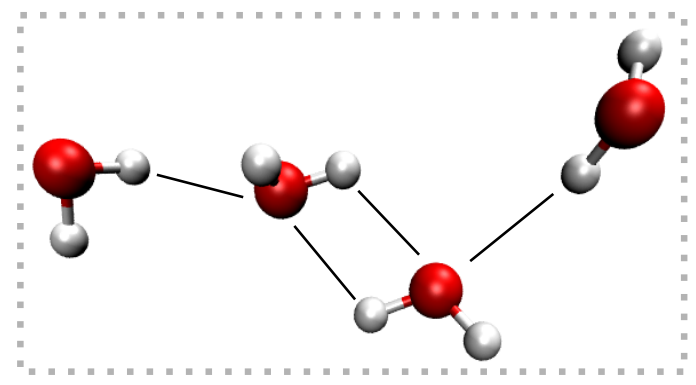

Figure S4: Atomic structures of water clusters obtained from DPMD simulations of supercritical water at $(773 \mathrm{~K}, 250 \mathrm{bar})$. HBs are represented by continuous black lines, and they have angular distortion larger than the angular cutoff imposed by the Luzar-Chandler HB criteria. Panels (a) and (b) contain dimers with cyclic and bifurcated HBs, respectively. Panels (c) and (d) contain a trimer and a tetramer with cyclic HB, respectively.

Table S3: Averaged number of HB, fraction of percolating configuration, and cluster analysis in DPMD simulation at $733 \mathrm{~K}$

\begin{tabular}{cccccc} 
& $\begin{array}{c}\text { Luzar- } \\
\text { Chandler }^{26}\end{array}$ & $\begin{array}{c}\text { Todorova } \\
\text { et al. }\end{array}$ & Luzar $^{29}$ & $\begin{array}{c}\text { Kuo- } \\
\text { Mundy }^{30}\end{array}$ & $\begin{array}{c}\text { Wernet } \\
\text { et al. }^{31}\end{array}$ \\
\hline \hline$N_{H B}$ & 0.4 & 0.5 & 0.4 & 0.3 & 0.3 \\
$f_{p}$ & $0 \%$ & $0 \%$ & $0 \%$ & $0 \%$ & $0 \%$ \\
\hline monomer & $63 \%$ & $61 \%$ & $68 \%$ & $76 \%$ & $74 \%$ \\
dimer & $20 \%$ & $20 \%$ & $19 \%$ & $16 \%$ & $17 \%$ \\
trimer & $9 \%$ & $9 \%$ & $8 \%$ & $5 \%$ & $6 \%$ \\
tetramer & $4 \%$ & $5 \%$ & $3 \%$ & $2 \%$ & $2 \%$ \\
pentamer & $2 \%$ & $2 \%$ & $1 \%$ & $1 \%$ & $1 \%$ \\
hexamer & $1 \%$ & $1 \%$ & $1 \%$ & $0 \%$ & $0 \%$ \\
heptamer & $1 \%$ & $1 \%$ & $0 \%$ & $0 \%$ & $0 \%$ \\
octamer & $0 \%$ & $0 \%$ & $0 \%$ & $0 \%$ & $0 \%$ \\
\hline \hline
\end{tabular}




\section{Oxygen-Oxygen Pair-Correlation Functions}

We computed the oxygen-oxygen pair-correlation functions (denoted $g_{\mathrm{OO}}(r)$ ) at $T=553 \mathrm{~K}$ (with percolating $\mathrm{HB}$ network) and $T=733 \mathrm{~K}$ (with non-percolating HB network) under $P=250$ bar using our DPMD trajectories. These $g_{\mathrm{OO}}(r)$ are compared with those from experimental assignments based on neutron diffraction. ${ }^{32}$ In these experimental assignments, each $g_{\mathrm{OO}}(r)$ was obtained using empirical potential structure refinement (EPSR) technique, ${ }^{33,34}$ which modifies the SPC/E force field ${ }^{35}$ to fit the experimentally probed interference differential cross-sections for supercritical water at percolating and non-percolating conditions.

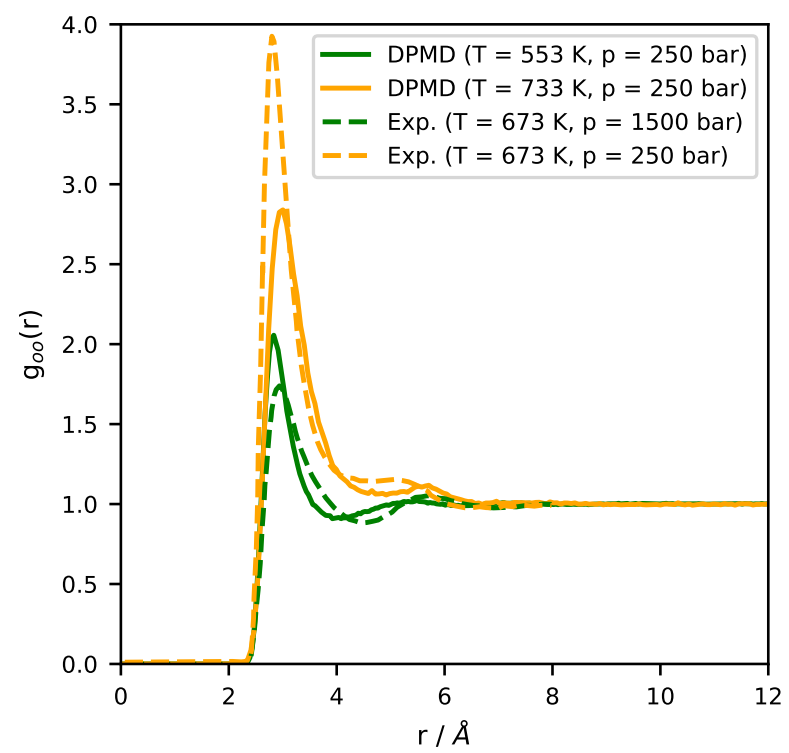

Figure S5: Oxygen-oxygen paircorrelation functions of water predicted by DPMD (continuous lines) and experiment/EPSR assignments ${ }^{32}$ (dashed lines).

In Figure S5, we compare $g_{\mathrm{OO}}(r)$ obtained from our DPMD simulations with those from experiment/EPSR assignments. Although the thermodynamic conditions of these experiments and our simulations differ, DPMD and experimental densities are close when comparing within percolating $\left(\approx 0.86 \mathrm{~g} / \mathrm{cm}^{3}\right.$ for DPMD at $553 \mathrm{~K}$ and 250 bar vs. $\approx 0.75 \mathrm{~g} / \mathrm{cm}^{3}$ for experiment at $673 \mathrm{~K}$ and $1500 \mathrm{bar})$ and non-percolating $\left(\approx 0.12 \mathrm{~g} / \mathrm{cm}^{3}\right.$ for DPMD at $733 \mathrm{~K}$ and 250 bar vs. $\approx 0.12 \mathrm{~g} / \mathrm{cm}^{3}$ for experiment at $673 \mathrm{~K}$ and $250 \mathrm{bar}$ ) conditions, allowing a qualitative comparison. In this comparison, we found consistent qualitative change when 
going from a percolating HB network to a non-percolating condition. More specifically, we observed a significant enhancement in the height of the first peak $(r=2.5-3.5 \AA)$ as well as the emergence of a plateaued shoulder from the originally oscillatory structure within $(r=3.5-6.0 \AA)$.

When the HB network is percolating, $g_{\mathrm{OO}}(r)$ from our DPMD behaves similarly as a liquid-like fluid with the presence of a second coordination shell separated by a minimum from the first coordination shell. Although weak, we observed features associated to intermediate range order (IRO). The same behavior can also be discerned in the $g_{\mathrm{OO}}(r)$ from the experiment/EPSR assignment. The sharper first peak in the $g_{\mathrm{OO}}(r)$ of our DPMD is a consequence of the lower temperature of the DPMD simulation relative to experiment. Both $g_{\mathrm{OO}}(r)$ from DPMD and experiment/EPSR are consistent with the presence of percolating HB networks as discussed in Tables S2-S3 and in experiment/EPSR assignment by Soper and coworkers. ${ }^{32}$

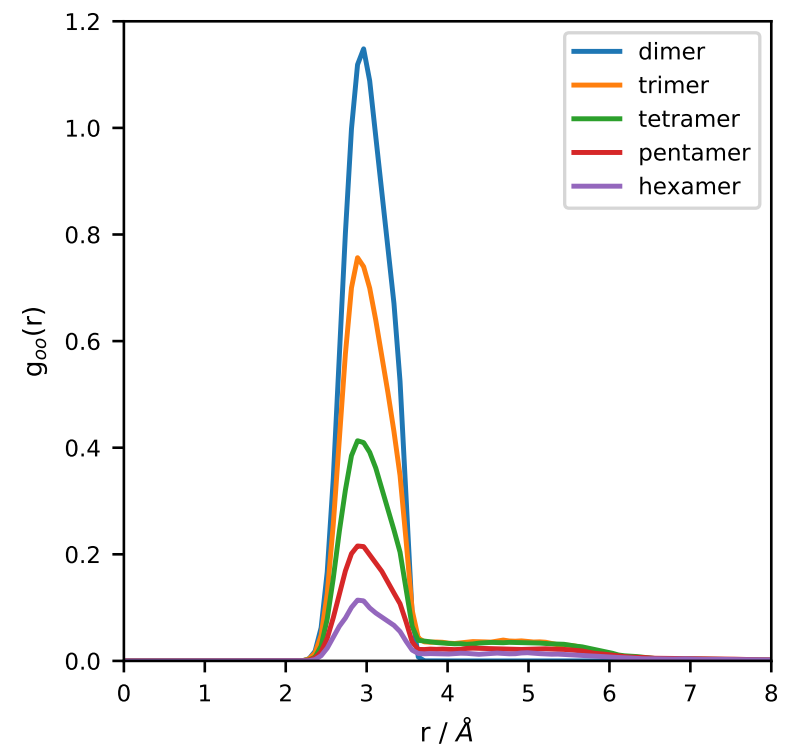

Figure S6: Cluster-size-resolved oxygen-oxygen pair-correlation functions $\left(g_{\mathrm{OO}}(r)\right)$. Only pair correlations involving water molecules with the targeted cluster size were included.

When the HB network is non-percolating, the DPMD simulation and experiment/EPSR assignment have $g_{\mathrm{OO}}(r)$ similar to those typically observed in real gases. Real gases have pair-correlation functions characterized by a sharp first peak (short range order, SRO) and absence of IRO (no second shell). For both our DPMD simulation and the experi- 
ment/EPSR assignment, the first peak of $g_{\mathrm{OO}}(r)$ shows a well defined plateaued-shoulder

for $r \approx 3.5-6.0 \AA$, after which $g_{\mathrm{OO}}(r)$ decays to one (i.e., no correlation). The plateauedshoulder originates from the presence of trimers and larger clusters in gas-like supercritical water as shown in the cluster-size-resolved $g_{\mathrm{OO}}(r)$ in Figure $\mathrm{S} 6{ }^{1}$

\section{Vibrational Density of States}

The vibrational density of states (VDoS) is computed from the spectrum of the velocity autocorrelation function. To eliminate the translational motions of each $\mathrm{H}_{2} \mathrm{O}$ from the spectra, we refer the molecular motions to their individual centers of mass. In order to assess the role of the librational/rotational modes, we also compute the VDoS of pure bending/stretching modes by projecting the molecular velocities in the center of mass frame of each molecule onto bending and stretching modes. For the ease for visual comparison, we normalize the VDoS based on the number of modes associated with $\left(\mathrm{H}_{2} \mathrm{O}\right)_{512}$.

\section{Widom and Frenkel lines}

In the supercritical (SC) region, the correlation length $\xi$ for thermodynamic fluctuations diverges at the critical point $(\mathrm{CP}),{ }^{36}$ and near the $\mathrm{CP}$ a critical behavior is observed for a number of response functions ruled by the second derivatives of the Gibbs thermodynamic potential, which are experimentally seen to go through extrema and show proportionality to a power function of $\xi$. This is also the case for density fluctuations. ${ }^{37}$

Such extrema reside on a set of loci - named "Widom lines" after Benjamin Widom that merge asymptotically into a single curve when approaching the liquid-gas $\mathrm{CP}$ and get more and more distant from one another as one goes several dozen percent away from it.

Though Widom lines signaling no $1^{\text {st }}$-order transition, they are nonetheless thought to cause a partition of the supercritical region into sub-regions characterized by different regimes

\footnotetext{
${ }^{1}$ Water clusters were identified using the Luzar-Chandler HB definition.
} 
reminiscent of their parent non-critical phases. Indeed, the difference of most thermodynamic properties between two fluid states residing inside and outside the SC region, respectively, in proximity of its borders, can be much smaller than the one observed in getting across a Widom line.

Alternatively, Brazhkin et al ${ }^{37}$ introduced a dynamic (rather than thermodynamic) criterion, based on the assumption that in a liquid, as temperature is increased, the relaxation time $\tau^{*}$ between two particle jumps eventually becomes comparable to the period $\tau_{0}$ of particle vibrations around a local equilibrium position, thus making the time-ruled discrimination between vibrational and hopping behavior disappear. When $\tau^{*}<\tau_{0}$, a transition to the ballistic-collision diffusion regime (typical of gases) occurs. Therefore, the condition $\frac{\tau *}{\tau^{0}} \sim 1$ serves as an effective dynamic crossover between a rigid-liquid regime and a quasigas soft-fluid one, especially in terms of shear resistance. This condition defines the so-called "Frenkel line" (after Yakov Frenkel), which is considered sort of "true" Widom line, in the proximity of which also the thermodynamic functions exhibit specific features, basically due to the disappearance of transverse-phonon type excitations in the short-wavelength part of the spectrum as temperature and pressure increase.

Being not formally related to the liquid-gas transition, the Frenkel line has a universal nature, since it can exist for systems in which the liquid-vapor coexistence curve and the CP are absent (such as some colloidal systems).

The Frenkel dynamic crossover can be recovered via the velocity autocorrelation function, since a transition from an oscillatory/decaying behavior (typical of liquids and solids near melting) to a monotonic decay (typical of gases) should be observed in passing from the liquid-like to the quasigas-like region. ${ }^{38}$

An alternative route is monitoring the diffusion coefficient. Indeed, being the average time of a jump to a distance equal to the interparticle spacing, in a random-walk model $\tau^{*}$ is related to the diffusion coefficient $D$ for the liquid like $\tau^{*} \sim \frac{a^{2}}{6 D}$, where $a$ is the shortest average interparticle distance. ${ }^{37}$ Consequently, the condition $\frac{\tau *}{\tau^{0}} \sim 1$ can be rewritten as 
$D \sim \frac{a^{2}}{6 \tau_{0}}$

At moderate pressures $\left(p<5-10 p_{c}\right)$, the compression of the fluid in the region of quasigas density mainly occurs due to a decrease in the free volume, and $\tau_{0}$ changes only slightly. The magnitude of $a^{2}$ also changes insignificantly over such range of pressures, which means that the locus of constant diffusion coefficients can be selected as the conditional line that separates a rigid liquid from a soft quasigas fluid. Across this dynamic line the temperature dependence of the diffusion coefficient - as well as of $\tau^{*}$ itself - changes from exponential to power-law in $T$, as confirmed via TIP4P/2005 MD simulation. ${ }^{39}$ As shown in Figure S7, the experiment monitored the behavior of SC water across the Widom and Frenkel lines along an isobar close to the liquid-gas CP.

\section{References}

(1) Granroth, G. E.; Kolesnikov, A. I.; Sherline, T. E.; Clancy, J. P.; Ross, K. A.; Ruff, J. P. C.; Gaulin, B. D.; Nagler, S. E. SEQUOIA: A Newly Operating Chopper Spectrometer at the SNS. Journal of Physics: Conference Series 2010, 251, 012058.

(2) Senesi, R.; Flammini, D.; Kolesnikov, A. I.; Murray, E. D.; Galli, G.; Andreani, C. The quantum nature of the $\mathrm{OH}$ stretching mode in ice and water probed by neutron scattering experiments. The Journal of Chemical Physics 2013, 139, 074504.

(3) Ricci, M. A.; Nardone, M.; Fontana, A.; Andreani, C.; Hahn, W. Light and neutron scattering studies of the $\mathrm{OH}$ stretching band in liquid and supercritical water. The Journal of Chemical Physics 1998, 108, 450-454.

(4) Andreani, C.; Colognesi, D.; Filabozzi, A.; Pace, E.; Zoppi, M. Deep inelastic neutron scattering from fluid para- and orthohydrogen. Journal of Physics: Condensed Matter 1998, 10, 7091.

(5) Andreani, C.; Filabozzi, A.; Nardone, M.; Ricci, F. P.; Mayers, J. Quantum and classical 


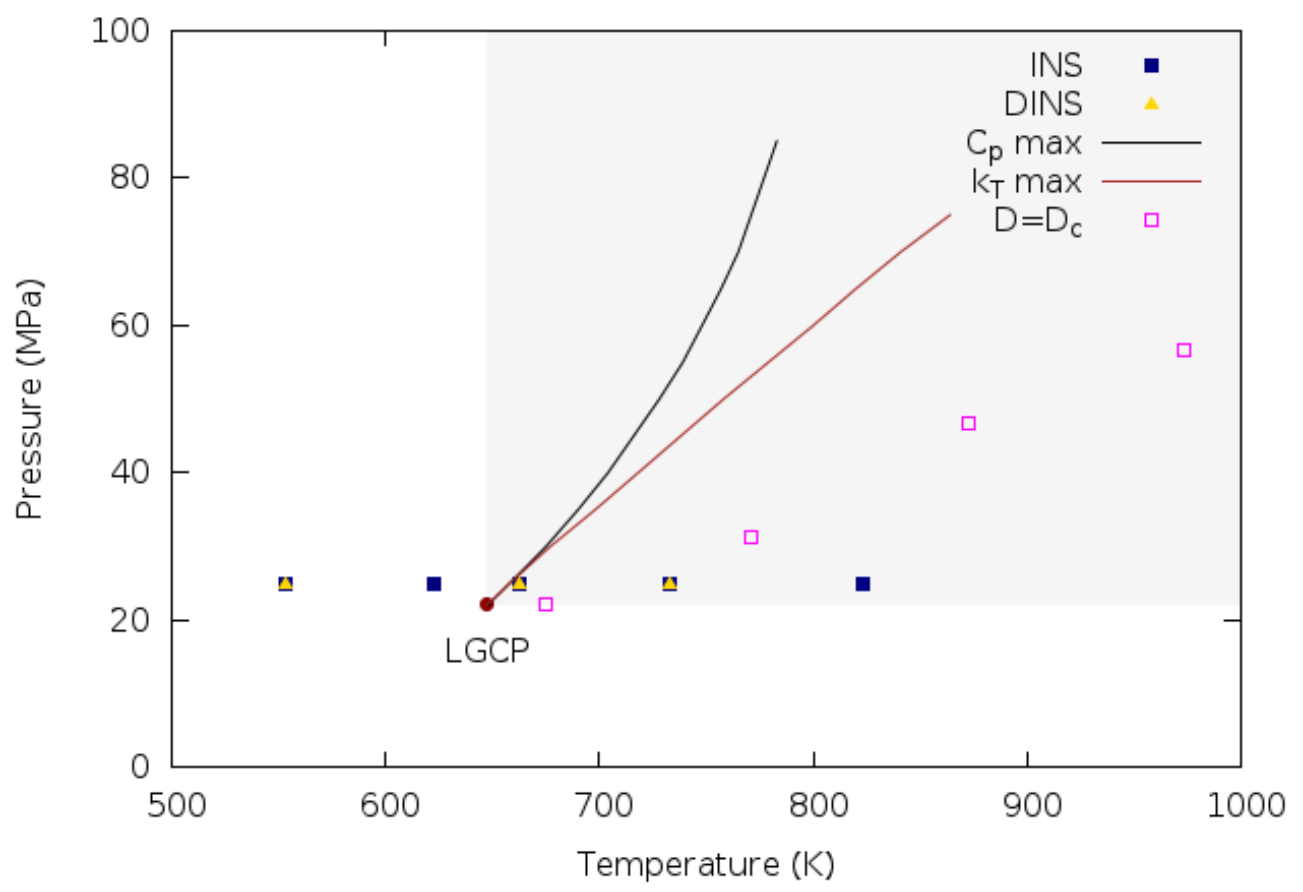

Figure S7: A 2D thermodynamic representation of the set of DINS measuments at VESUVIO (filled yellow triangles) and INS determinations at SEQUOIA (filled blue squares) with respect to a couple Widom lines emanating from the liquid-gas critical point (LGCP) and the Frenkel line. The locus of $C_{p}$ maxima (black curve) was reconstructed out of NIST data (http://webbook.nist.gov/chemistry/fluid/), and that of $k_{T}$ maxima (brown curve) out of ThermoC data (http://thermoc.uni-koeln.de/thermoc/) within the IAPWS-95 model. The locus of constant self-diffusion coefficients (open magenta squares) was reconstructed out of experimental NMR data. ${ }^{40}$ The grey box delimits the $\mathrm{SC}$ region and is intended as a guide for the eye. 
behavior of single-particle dynamics in dense liquid ${ }^{4}$ He. Phys. Rev. B 1994, 50, 1274412746.

(6) Andreani, C.; Bosi, P.; Sacchetti, F.; Loong, C. K. Absolute measurements of the stretching mode density of states in polycrystalline ice Ih. The Journal of Chemical Physics 1985, 83, 750-753.

(7) Tardocchi, M.; Pietropaolo, A.; Andreani, C.; Bracco, A.; D’Angelo, A.; Gorini, G.; Imberti, S.; Senesi, R.; Rhodes, N. J.; Schooneveld, E. M. Cadmium-Zinc-Telluride photon detector for epithermal neutron spectroscopy-pulse height response characterisation. Nuclear Instruments and Methods in Physics Research Section A 2004, 526, $477-492$.

(8) Schooneveld, E. M.; Mayers, J.; Rhodes, N. J.; Pietropaolo, A.; Andreani, C.; Senesi, R.; Gorini, G.; Perelli-Cippo, E.; Tardocchi, M. Foil cycling technique for the VESUVIO spectrometer operating in the resonance detector configuration. Review Scientific Instruments 2006, 77, 5103.

(9) Andreani, C.; Pietropaolo, A.; Senesi, R.; Gorini, G.; Tardocchi, M.; Bracco, A.; Rhodes, N.; Schooneveld, E. Electron-volt spectroscopy at a pulsed neutron source using a resonance detector technique. Nuclear Instruments and Methods in Physics Research Section A 2002, 481, 509-520.

(10) Rebai, M.; Andreani, C.; Fazzi, A.; Frost, C. D.; Giacomelli, L.; Gorini, G.; Milani, E.; Perelli Cippo, E.; Pietropaolo, A.; Prestopino, G. et al. Fission diamond detector tests at the ISIS spallation neutron source. Nuclear Physics B Proceedings Supplements 2011, 215, 313-315.

(11) Imberti, S.; Andreani, C.; Garbuio, V.; Gorini, G.; Pietropaolo, A.; Senesi, R.; Tardocchi, M. Resolution of the VESUVIO spectrometer for High-energy Inelastic Neutron 
Scattering experiments. Nuclear Instruments and Methods in Physics Research Section A 2005, 522, 463.

(12) Andreani, C.; D’Angelo, A.; Gorini, G.; Imberti, S.; Pietropaolo, A.; Rhodes, N. J.; Schooneveld, E. M.; Senesi, R.; Tardocchi, M. CdZnTe $\gamma$ detector for deep inelastic neutron scattering on the VESUVIO spectrometer. Applied Physics A: Materials Science $\&$ Processing 2004, 78, 903-913.

(13) Andreani, C.; Krzystyniak, M.; Romanelli, G.; Senesi, R.; Fernandez-Alonso, F. Electron-volt neutron spectroscopy: beyond fundamental systems. Advances in Physics 2017, 66, 1-73.

(14) Giannozzi, P.; Baroni, S.; Bonini, N.; Calandra, M.; Car, R.; Cavazzoni, C.; Ceresoli, D.; Chiarotti, G. L.; Cococcioni, M.; Dabo, I. et al. QUANTUM ESPRESSO: a modular and open-source software project for quantum simulations of materials. J. Phys.: Condens. Matter 2009, 21, 395502.

(15) Giannozzi, P.; Andreussi, O.; Brumme, T.; Bunau, O.; Nardelli, M. B.; Calandra, M.; Car, R.; Cavazzoni, C.; D Ceresoli,; Cococcioni, M. et al. Advanced capabilities for materials modelling with Quantum ESPRESSO. J. Phys.: Condens. Matter 2017, 29, 465901.

(16) Sun, J.; Ruzsinszky, A.; Perdew, J. P. Strongly Constrained and Appropriately Normed Semilocal Density Functional. Phys. Rev. Lett. 2015, 115, 036402.

(17) Sun, J.; Remsing, R. C.; Zhang, Y.; Sun, Z.; Ruzsinszky, A.; Peng, H.; Yang, Z.; Paul, A.; Waghmare, U.; Wu, X. et al. Accurate first-principles structures and energies of diversely bonded systems from an efficient density functional. Nat Chem 2016, 8, 831-836.

(18) Chen, M.; Ko, H.-Y.; Remsing, R. C.; Andrade, M. F. C.; Santra, B.; Sun, Z.; Sell- 
oni, A.; Car, R.; Klein, M. L.; Perdew, J. P. et al. Ab initio theory and modeling of water. Proc. Natl. Acad. Sci. U.S.A. 2017, 114, 10846-10851.

(19) Hamann, D. R.; Schlüter, M.; Chiang, C. Norm-Conserving Pseudopotentials. Phys. Rev. Lett. 1979, 43, 1494.

(20) Vanderbilt, D. Optimally smooth norm-conserving pseudopotentials. Phys. Rev. B 1985, 32, 8412.

(21) Perdew, J. P.; Burke, K.; Ernzerhof, M. Generalized Gradient Approximation Made Simple. Phys. Rev. Lett. 1996, 7r, 3865-3868.

(22) Gygi, F. Architecture of Qbox: A Scalable First-principles Molecular Dynamics Code. IBM J. RES. \& DEV. 2008, 52, 137.

(23) Jurečka, P.; Šponer, J.; Černý, J.; Hobza, P. Benchmark database of accurate (MP2 and $\operatorname{CCSD}(\mathrm{T})$ complete basis set limit) interaction energies of small model complexes, DNA base pairs, and amino acid pairs. Phys. Chem. Chem. Phys. 2006, 8, 1985-1993.

(24) Martyna, G. J.; Klein, M. L.; Tuckerman, M. Nosé-Hoover chains: the canonical ensemble via continuous dynamics. J. Chem. Phys. 1992, 97, 2635.

(25) Parrinello, M.; Rahman, A. Crystal Structure and Pair Potentials: A MolecularDynamics Study. Phys. Rev. Lett. 1980, 45, 1196.

(26) Luzar, A.; Chandler, D. Hydrogen-bond kinetics in liquid water. Nature 1996, 379, 55.

(27) Strong, S. E.; Shi, L.; Skinner, J. L. Percolation in supercritical water: Do the Widom and percolation lines coincide? J. Chem. Phys. 2018, 149, 084504.

(28) Todorova, T.; Seitsonen, A. P.; Hutter, J.; Kuo, I.-F. W.; Mundy, C. J. Molecular Dynamics Simulation of Liquid Water: Hybrid Density Functionals. J. Phys. Chem. B 2006, 110, 3685-3691. 
(29) Luzar, A. Resolving the hydrogen bond dynamics conundrum. J. Chem. Phys. 2000, 113, 10663-10675.

(30) Kuo, I.-F. W.; Mundy, C. J. An ab Initio Molecular Dynamics Study of the Aqueous Liquid-Vapor Interface. Science 2004, 303, 658-660.

(31) Wernet, P.; Nordlund, D.; Bergmann, U.; Cavalleri, M.; Odelius, M.; Ogasawara, H.; Näslund, L. A.; Hirsch, T. K.; Ojamäe, L.; Glatzel, P. et al. The Structure of the First Coordination Shell in Liquid Water. Science 2004, 304, 995-999.

(32) Bernabei, M.; Botti, A.; Bruni, F.; Ricci, M. A.; Soper, A. K. Percolation and threedimensional structure of supercritical water. Phys. Rev. E 2008, 78, 021505.

(33) Soper, A. K. Tests of the empirical potential structure refinement method and a new method of application to neutron diffraction data on water. Mol. Phys. 2001, 99, 15031516.

(34) Soper, A. K. Partial structure factors from disordered materials diffraction data: An approach using empirical potential structure refinement. Phys. Rev. B 2005, 72, 104204.

(35) Berendsen, H. J. C.; Grigera, J. R.; Straatsma, T. P. The missing term in effective pair potentials. J. Phys. Chem. 1987, 91, 6269-6271.

(36) Stanley, H. E. Introduction to Phase Transitions and Critical Phenomena, by H Eugene Stanley, pp. 336. Foreword by H Eugene Stanley. Oxford University Press, Jul 1987. ISBN-10: 0195053168. ISBN-13: 9780195053166; 1987; p 336.

(37) Brazhkin, V. V.; Lyapin, A. G.; Ryzhov, V. N.; Trachenko, K.; Fomin, Y. D.; Tsiok, E. N. Where is the supercritical fluid on the phase diagram? Physics Uspekhi 2012, 55, 1061-1079. 
(38) Brazhkin, V. V.; Fomin, Y. D.; Lyapin, A. G.; Ryzhov, V. N.; Tsiok, E. N.; Trachenko, K. "Liquid-Gas" Transition in the Supercritical Region: Fundamental Changes in the Particle Dynamics. Phys. Rev. Lett. 2013, 111, 145901.

(39) Gallo, P.; Corradini, D.; Rovere, M. Widom line and dynamical crossovers as routes to understand supercritical water. Nature Communications 2014, 5, 5806.

(40) Lamb, W. E. Capture of Neutrons by Atoms in a Crystal. Phys Rev 1939, 55, 190-197. 